\title{
Overview and Status of the Lunar Detection of Cosmic Particles with LOFAR
}

T. Winchen ${ }^{1 *}$ A. Bonardi ${ }^{2}$, S. Buitink ${ }^{1}$, A. Corstanje ${ }^{2}$, H. Falcke ${ }^{2,3,4}$, B. M. Hare ${ }^{5}$, J. R. Hörandel ${ }^{2,3}$, P. Mitra ${ }^{1}$, K. Mulrey ${ }^{1}$, A. Nelles ${ }^{2,6}$, J. P. Rachen ${ }^{2}$, L. Rossetto ${ }^{2}$, P. Schellart ${ }^{2,7}$, O. Scholten ${ }^{5,8}$, S. ter Veen ${ }^{2,4}$, S. Thoudam ${ }^{2,9}$, T. N. G. Trinh ${ }^{5}$

${ }^{1}$ Astrophysical Institute, Vrije Universiteit Brussel, Pleinlaan 2, 1050 Brussels, Belgium,

${ }^{2}$ Department of Astrophysics/IMAPP, Radboud University, P.O. Box 9010, 6500 GL Nijmegen, The Netherlands,

${ }^{3}$ NIKHEF, Science Park Amsterdam, 1098 XG Amsterdam, The Netherlands,

${ }^{4}$ Netherlands Institute of Radio Astronomy (ASTRON), Postbus 2, 7990 AA Dwingeloo, The Netherlands,

${ }^{5}$ KVI-CART, University Groningen, P.O. Box 72, 9700 AB Groningen,

${ }^{6}$ Department of Physics and Astronomy, University of California Irvine, Irvine, CA 92697-4575, USA,

${ }^{7}$ Department of Astrophysical Sciences, Princeton University, Princeton, NJ 08544, USA,

${ }^{8}$ Interuniversity Institute for High-Energy, Vrije Universiteit Brussel, Pleinlaan 2, 1050 Brussels, Belgium,

${ }^{9}$ Department of Physics and Electrical Engineering, Linnéuniversitetet, 35195 Växjö, Sweden

E-mail: tobias.winchenerwth-aachen. de

\begin{abstract}
When a cosmic particle hits matter it produces radio emission via the Askaryan effect. This allows to use Earth's moon as detector for cosmic particles by searching for these ns-pulses with radio telescopes. This technique potentially increases the available collective area by several orders of magnitude compared to current experiments. The LOw Frequency ARray (LOFAR) is the largest radio telescope operating in the optimum frequency regime for corresponding searches. In this contribution, we report on the design and status of the implementation of the lunar detection mode at LOFAR.
\end{abstract}

35th International Cosmic Ray Conference - ICRC2017

10-20 July, 2017

Bexco, Busan, Korea

${ }^{*}$ Speaker. 


\section{Introduction}

The low intensity of the cosmic-ray flux at the highest energies (UHECR) is one of the major challenges in answering the long standing open questions about their origin, acceleration, and composition (e.g. [1]). The same challenge arises in testing specific theories on super heavy dark matter [2,3] and grand-unification [4] that predict an even lower flux of neutrinos with energies beyond the Zetta-eV scale. Even the largest currently operating cosmic-ray experiments [5, 6] detect only dozens of the highest energy particles per year, but cover already areas on the scale of small nations. It is thus doubtful, that experiments with an increase of more than an order of magnitude in sensitive volume can be realized on Earth.

Searching beyond Earth's atmosphere, a possible detector volume may be provided by Earth's moon, as particle interactions in the lunar rock emit a radio signal emitted by the Askaryan effect [7]. These radio signals can be recorded by an antenna array on the Moon's surface, or possibly cheaper with by radio-observatories on Earth [8]. Previous searches with Earth-bound radio telescopes have established only upper limits limits on the $\mathrm{ZeV}$-scale neutrino flux, and have not been sensitive enough to constrain underlying production models or to observe UHECR [9-13].

An important limiting factor for these experiments is the frequency range of the used telescopes. While at $\mathrm{GHz}$ frequencies the expected pulse amplitude reaches a maximum, the pulse cannot escape the moon on most of its surface. The high frequencies are strongly beamed in direction of the Cherenkov angle, while the lower frequencies are emitted in a broader cone. As the critical angle of total internal reflection is identical to the Cherenkov angle, only radiation which strikes the surface at an acute angle with respect to the surface normal is emitted by the Moon. The lower frequencies can thus reach Earth at a wider range of impact angles of the primary particle than the higher frequencies. Also, as the lunar rock is more transparent to lower frequencies, the diminished signal amplitude is greatly outweighed by the increase in effective detector volume. Together, this results in an improved sensitivity of searches with telescopes operating at lower frequencies compared to searches that used higher frequencies. The optimal frequency range for lunar observations thus includes frequencies just above approximately $100 \mathrm{MHz}$ [14].

\section{The LOw Frequency ARray}

The currently largest telescope covering the optimal frequency range for lunar detection of cosmic particles is the LOw Frequency ARray (LOFAR) [15]. As the first fully digital radio telescope, LOFAR contains no moveable dishes, but consists of fixed antenna arrays whose signals are directed into beams by digital processing.

The antennas arrays are grouped into multiple stations distributed throughout the Netherlands and with additional stations in France, Germany, Poland, the United Kingdom, and Sweden. Twenty-four of the stations are located in a dense core with an diameter of approximately $2 \mathrm{~km}$ in the Netherlands. The additional stations are distributed with increasing distances to this core yielding a maximum baseline of $1292 \mathrm{~km}$. However, for lunar particle detection only the core stations can be used, as the data has to be processed in realtime and only the core stations are connected sufficiently fast. 
Each core station is equipped with fields of 96 low-band antennas (LBAs) with a frequency range from 10-90 MHz and fields of 192 high-band antennas (HBAs) with a frequency range from 110-240 MHz. The signal received by the antennas is sampled in intervals of $5 \mathrm{~ns}$ and copied to a ring buffer of $5 \mathrm{~s}$ length at antenna level before being processed further. The signals of the individual omni-directional antennas are then filtered into sub-bands by a polyphase filter (PPF) and combined into a single beam of approximately $5^{\circ}$ width at $120 \mathrm{MHz}$ per station pointing towards a user-defined direction in the sky. A selection of sub-bands is then transmitted to a computing cluster for further processing as e.g. combining the station beams into smaller 'tied-array' beams and integrating the signal over longer time spans. Each station thus assumes the role of a single dish in a classical radio telescope.

\section{Lunar Detection of Cosmic Particles with LOFAR}

The lunar detection of cosmic particles with LOFAR faces several technical challenges. To maximize the effective area the signals of multiple stations have to be combined. As this reduces the size of the individual beams, multiple beams are required to cover the entire Moon. As each station, respectively beam, produces data with a rate of more than $6.4 \mathrm{Gbits}^{-1}$, analysis of the data in real-time is required as only selective storage of the data is possible. Signals originating from the moon are dispersed in the Earth's atmosphere. Triggering on the amplitude of a pulse thus requires correction for the dispersion. The pulse from a particle interaction is of only ns duration. By the PPF the time-resolution of the signal is reduced from $5 \mathrm{~ns}$ to only about $5 \mu$ in the individual subbands. Therefore, the inversion of the PPF is required for the lunar detection of paticles. However, the inversion is not a loss-less procedure. We therefore use the recovered ns resolution signal only to trigger the read-out of the Transient-Buffer-Boards containing the original ADC traces. The offline analysis will thus not be limited by the accuracy of the PPF inversion which may produce artefacts, but all realtime-processing, i.e. beamforming, PPF inversion, ionospheric de-dispersion, and triggering has to be done within $5 \mathrm{~s}$.

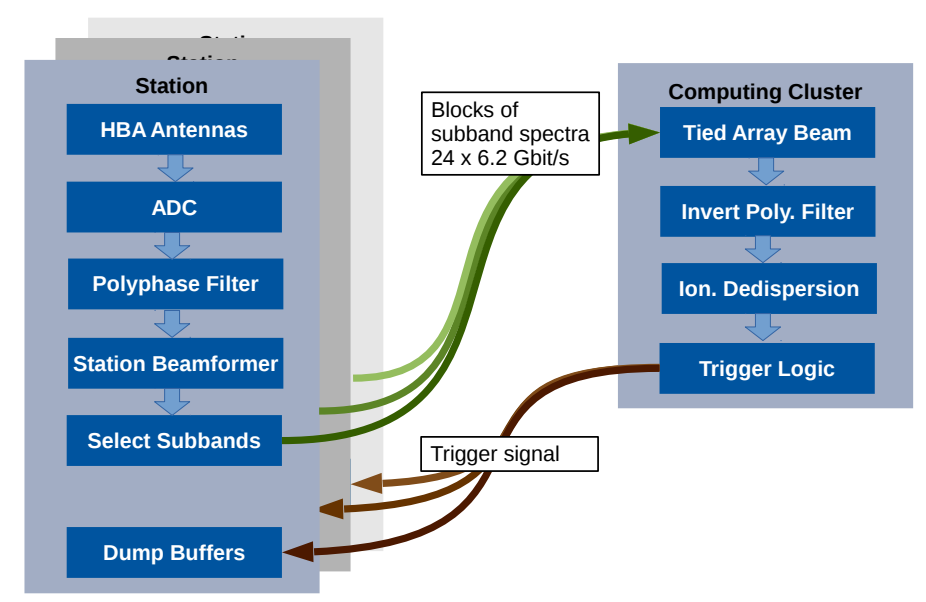

Figure 1: Overview of the online data analysis processing steps for the detection of ns-pulses with LOFAR [16]. 
The resulting sequence of the data processing steps necessary for the lunar detection of cosmic particles with LOFAR is sketched in figure 1. After transfer to the computing cluster, the station beams, each covering the full moon, will first be combined into multiple smaller beams, each focussed on a small fraction of the moon. The signal in ns resolution within each beam will then be recovered by inversion of the PPF, before the signal will be de-dispersed and eventually a trigger will be sent.

Prototypes for all necessary processing steps before the trigger have been developed. For the trigger we for now assume only a simple threshold excess. The real-time signal processing will require several thousand GFLOPs ${ }^{-1}$ of computing power per beam. Here, inversion of the PPF is the most costly operation [17]. This required processing power is not available on the regular LOFAR processing machines, but the adjacent DRAGNET CPU/GPU cluster [18] designed for pulsar searches. However, the network connection limits the amount of data that can be processed in realtime on DRAGNET. Therefore a subset of 5-7 stations has to be selected for online analysis.

\section{Station Selection and Beam Properties}

The choice of LOFAR stations defines the properties of the individual beams and herby the quality of the received signal. For lunar detection of cosmic particles, the following beam properties are desirable. As we can process 48 beams at maximum with the available computing power, the beam-size has to be symmetrical and of approx. $0.5^{\circ}$ size to be able to cover the full moon.

As only 5 out of 48 substations can be used, 1712304 possible combinations exists which are too many to study individually in detail. However, based on the station position and orientation several combinations can be excluded without detailed simulation.

For LOFAR, the beam-size at wavelength $\lambda$ can be estimated to

$$
\Theta_{\mathrm{FWHM}}=.8 \frac{\lambda}{L}
$$

based on the maximum baseline between the stations $L$ [15]. We consequently do not consider combinations with a maximum baseline below $1000 \mathrm{~m}$ and above $2000 \mathrm{~m}$.

To achieve symmetry, the selected stations have to be arranged in a circular pattern as otherwise the beam-shape is also elongated. To quantify this behavior, we use the length of the resultant $R=\frac{1}{N}\left\|\sum_{i} \vec{v}_{i}\right\|$ from the ensemble of station positions relative to the geometrical center of the subset of stations (cf. e.g. [19]).

In addition, the included baselines and station orientations should be uniformly distributed to minimize sidelobes. We again use the length of the resultant calculated from the station orientations to quantify the distribution of orientations. To quantify the uniformness of the distribution of baselines we use the standard Kolmogorov distance.

Using these variables as preselector, we calculated the beams of few selected combinations to find a suitable combination of stations. Figure 2 (a) shows the beam of a combination of stations that is acceptable in the maximum beam-size and has an above average uniformity of distribution of baselines, but has a directed distribution of beam-lines. Consequently the gain patterns are also directed, and several sidelobes are visible. The example beam shown in figure 2 (b) has good values also for the directions of the stations and beam-lines, resulting in asymmetric beam with few sidelobes. The corresponding stations are marked in the map of HBA stations shown in figure 3 . 

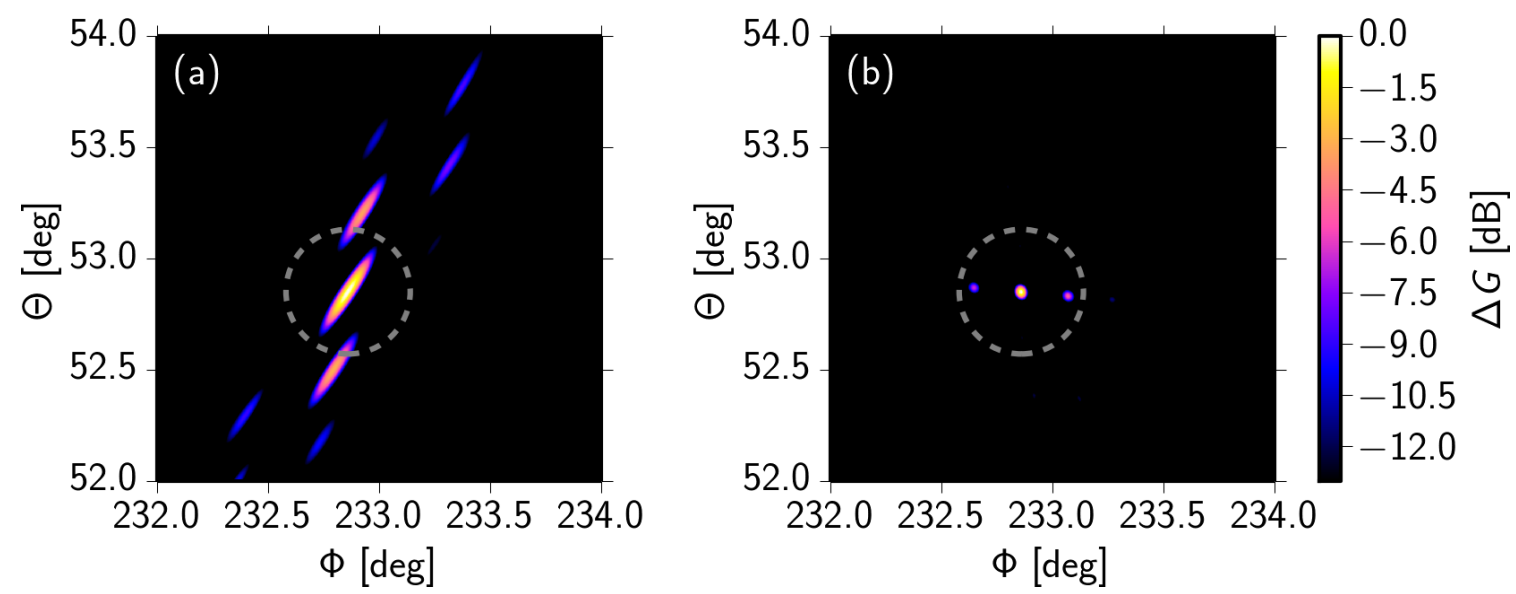

Figure 2: Example beams from different combinations of stations as indicated in figure 3. The dashed line marks the size of Earth's moon.



Figure 3: Map of the LOFAR core based on data by OpenStreetMap [20]. LOFAR HBA fields are marked with squares. Red markers correspond to station selection as in beam example fig. 2 (a), blue markers correspond to station selection as in beam example fig. 2 (b). Stations marked in black are not used in the examples.

The preselected combinations still have a large diversity in the properties of the beam and sidelobes. The final choice of stations will depend on the results of simulations of different trigger possibilities, with the achievable sensitivity as the deciding metric.

\section{Expected Sensitivity}

Currently, the best estimate of the expected sensitivity to cosmic particles with lunar obser- 


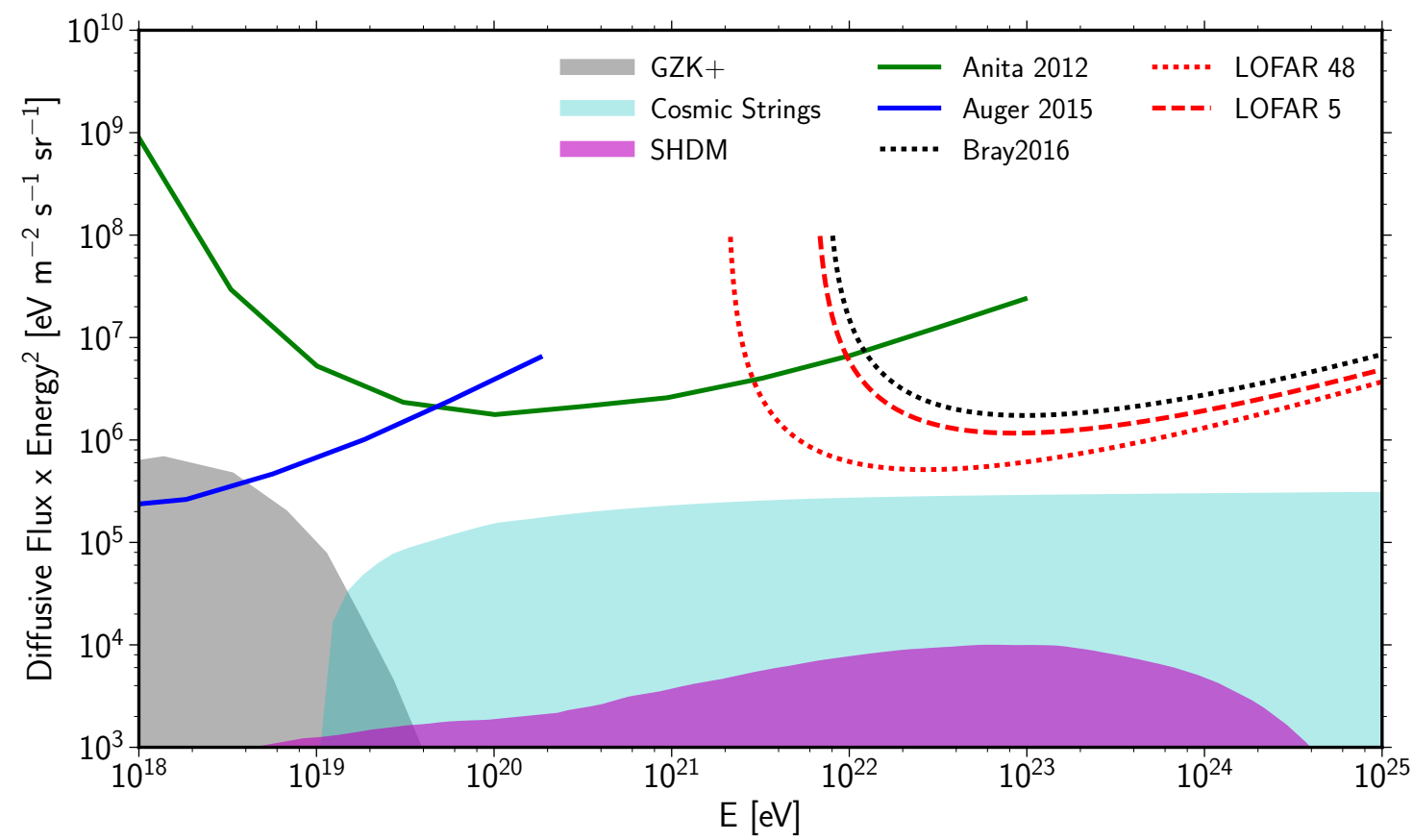

Figure 4: Existing limits (solid lines) on the flux of ultra high energetic neutrinos of previous observations $[21,22]$ and estimated limits for future observations (dashed and dotted lines). Model predictions for the flux of neutrinos from interaction of cosmic rays with background photons (GZK+) [23], cosmic strings [24], and super heavy dark matter (SHDM) [3] are marked as shaded areas.

vations is obtained following the procedure used in [25, 26]. Here, analytical approximations for multiple experiments are derived based on small angle approximations and constant transmission coefficients. While these approximations reproduce some features of early Monte Carlo simulations, the simplifications are valid only for large frequencies and may be in particular uncertain with the low observation frequencies used here. However, while the absolute scale of the sensitivity is uncertain, the approximation can be used to estimate the relevance of individual design choices.

In contrast to the revised calculation of the LOFAR sensitivity Bray 2016 [26], we here assume triggering on the coherently added polarizations and a trigger threshold $n_{\sigma}=6.4$ instead of $n_{\sigma}=12.4$ yielding a trigger rate of $1 \mathrm{~min}^{-1}$. Furthermore, the previous estimate was based on a bandwidth of $48 \mathrm{MHz}$, while now $78.125 \mathrm{MHz}$ can be used. The combined effect of these changes of parameters is shown assuming 5 and 48 substations in figure 4 for $200 \mathrm{~h}$ of observation. While the reduction of the total effective area to $A_{\text {eff }} \approx 2150 \mathrm{~m}$ for a moon elevation of $56^{\circ}$ significantly reduces the expected sensitivity to using the full LOFAR array, the improved bandwidth and trigger assumptions still yield an improvement compared to the previous estimate.

\section{Conclusion}

Using the moon as detector that is read-out by Earth based radio telescopes still provides a chance to significantly increase the sensitive volume to detect high energetic cosmic particles. 
However, conducting measurements with existing telescopes faces several technical challenges, as these instruments are not designed to operate on nano-second timescales. Nevertheless, observations with LOFAR, the currently most sensitive instrument, are currently in preparation. These observations can likely meet or exceed the previously estimated sensitivity.

\section{Acknowledgements}

The LOFAR cosmic ray key science project acknowledges funding from an Advanced Grant of the European Research Council (FP/2007-2013) / ERC Grant Agreement n. 227610. The project has also received funding from the European Research Council (ERC) under the European Union's Horizon 2020 research and innovation programme (grant agreement No 640130). We furthermore acknowledge financial support from FOM, (FOM-project 12PR304) and NWO (VENI grant 639041-130). LOFAR, the Low Frequency Array designed and constructed by ASTRON, has facilities in several countries, that are owned by various parties (each with their own funding sources), and that are collectively operated by the International LOFAR Telescope foundation under a joint scientific policy.

\section{References}

[1] K. Kotera and A. V. Olinto, Annual Review of Astronomy and Astrophysics 49, 119-153 (2011), arXiv:1101.4256 [astro-ph.HE].

[2] R. Aloisio, V. Berezinsky, and M. Kachelriess, Phys. Rev. D74, 023516 (2006), arXiv:a st roph/0604311 [astro-ph].

[3] R. Aloisio, S. Matarrese, and A. V. Olinto, JCAP 1508, 024 (2015), arXiv:1504 01319 [astro-ph.HE].

[4] P. Bhattacharjee, [AIP Conf. Proc.433,168(1998)] (1997) 10.1063/1.56108, arXiv:astro$\mathrm{ph} / 9803029$ [astro-ph].

[5] A. Aab et al., Nucl. Instrum. Meth. A798, 172-213 (2015), arXiv:1502. 01323 [astro-ph. IM] .

[6] H. Kawai et al., Nuclear Physics B - Proceedings Supplements 175, Proceedings of the XIV International Symposium on Very High Energy Cosmic Ray Interactions, 221-226 (2008).

[7] G. Askaryan, Sovjet Physics J.E.T.P 14, 441 (1962).

[8] R. D. Dagkesamanskij and I. M. Zheleznykh, Soviet Journal of Experimental and Theoretical Physics Letters 50, 259-261 (1989).

[9] S. Buitink et al., Astron. Astrophys. 521, A47 (2010), arXiv:1 004 . 0274 [astro-ph.HE] .

[10] T. H. Hankins, R. D. Ekers, and J. D. O'Sullivan, Monthly Notices of the Royal Astronomical Society 283, 1027-1030 (1996).

[11] C. W. James et al., Mon. Not. Roy. Astron. Soc. 410, 885-889 (2011), arXiv:0906. 3766 [astro-ph.HE].

[12] C. W. James et al., Mon. Not. Roy. Astron. Soc. 379, 1037-1041 (2007), arXiv:astroph/0702619 [astro-ph]. 
[13] J. D. Bray et al., Astropart. Phys. 65, 22-39 (2014), arXiv:1412. 4418 [astro-ph. IM] .

[14] O. Scholten et al., Astroparticle Physics 26, 219-229 (2006).

[15] M. P. van Haarlem et al., Astronomy and Astrophysics 556, A2 (2013), arXiv:1305. 3550 [astro-ph. IM].

[16] T. Winchen et al., EPJ WoC 135 (2017), arXiv:1609. 06590.

[17] T. Winchen et al., Accepted for publication in JPCS (2016), arXiv:1612. 06592.

[18] J. Hessels, DRAGNET: a high-speed, wide-angle camera for catching extreme astrophysical events, (Dec. 12, 2016) http://www.astron.nl/dragnet.

[19] K. V. Mardia, Statistics of directional data (Academic Press, London, 1972).

[20] OpenStreetMap contributors, OSM Version 0.6, (May 11, 2016) https : / / www . openstreetmap.? org.

[21] P. W. Gorham et al., Phys. Rev. D 85, 049901 (2012).

[22] A. Aab et al., Phys. Rev. D91, 092008 (2015), arXiv:1504.05397 [astro-ph.HE].

[23] E. Roulet et al., Journal of Cosmology and Astroparticle Physics 1, 028, 28 (2013), arXiv:1209. 4033 [astro-ph.HE].

[24] V. Berezinsky, E. Sabancilar, and A. Vilenkin, Phys. Rev. D84, 085006 (2011), arXiv:11 08. 2509 [astro-ph.CO].

[25] K. G. Gayley, R. L. Mutel, and T. R. Jaeger, Astrophys. J. 706, 1556-1570 (2009), arXiv:0 904 . 3389 [astro-ph.CO].

[26] J. Bray, Astroparticle Physics 77, 1-20 (2016), arXiv:1601.02980 [astro-ph. IM] . 